\title{
Locally preferred structures and many-body static correlations in viscous liquids
}

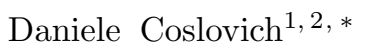 \\ ${ }^{1}$ Laboratoire Charles Coulomb UMR 5221, Université Montpellier II et CNRS, Montpellier, France \\ ${ }^{2}$ Institut für Theoretische Physik and CMS, Technische Universität Wien, Vienna, Austria
}

(Dated: October 22, 2019)

\begin{abstract}
We investigate the influence of static correlations beyond the pair level on the dynamics of selected model glass-formers. We compare the pair structure, angular distribution functions, and statistics of Voronoi polyhedra of two well-known Lennard-Jones mixtures as well as of the corresponding Weeks-Chandler-Andersen variants, in which the attractive part of the potential is truncated. By means of the Voronoi construction we identify the atomic arrangements corresponding to the locally preferred structures of the models. We find that the growth of domains formed by interconnected locally preferred structures signals the onset of the slow dynamics regime and allows to rationalize the different dynamic behaviors of the models. At low temperature, the spatial extension of the structurally correlated domains, evaluated at fixed relaxation time, increases with the fragility of the models and is systematically reduced by truncating the attractions. In view of these results, proper inclusion of many-body static correlations in theories of the glass transition appears crucial for the description of the dynamics of fragile glass-formers.
\end{abstract}

PACS numbers: 61.43.Fs, 61.20.Lc, 64.70.Pf, 61.20.Ja

\section{INTRODUCTION}

The question of whether the structure of a glass differs from that of the corresponding liquid is often rhetorically posed within the glass community. In fact, very small differences are observed in the static structure factor of a viscous liquid as it approaches the glass transition temperature $T_{g}$. By contrast, the viscosity and structural relaxation times increase by several orders of magnitude upon supercooling and the motion of the molecules in the liquid becomes increasingly cooperative and spatially heterogeneous.

At first glance, the small structural changes discernible at the level of pair correlations appear insufficient to explain the dramatic slowing down of the liquid and the non-trivial spatial correlations of the dynamics. A preliminary indication that this may not necessarily be the case already comes from the Mode Coupling theory (MCT) of the glass transition [1. The MCT predictions for the dynamic correlation functions are based uniquely on structural information, almost invariably the static structure factors of the liquid. Numerical solutions of the MCT equations for model liquids show that small variations of the pair correlation functions, which develop upon lowering the temperature, can produce significant effects on the dynamics and eventually lead to an ergodic-non-ergodic transition at some critical temperature $T_{\mathrm{MCT}}$ [2]. The generic predictions of the theory account rather well for experimental and numerical findings in weakly supercooled liquids [1, 3] (i.e., for $T \gtrsim T_{\mathrm{MCT}}$ ). The break-down of MCT at lower temperatures $\left(T_{g}<\right.$ $\left.T \lesssim T_{\mathrm{MCT}}\right)$, where the actual system remains effectively ergodic, most likely reflects the mean-field character of

\footnotetext{
* Email: daniele.coslovich@univ-montp2.fr
}

the theory [4 6] and its inability to describe activated transitions between metastable glassy states [7. Another delicate aspect that may affect the outcome of MCT is the exclusion of many-body correlations 8. Three-body static correlations have been shown [9] to impact the MCT solutions for a model of silica [10, but not the ones for the prototypical Kob-Andersen model 11. Interestingly, a study of a schematic version of the generalized MCT [12 14, which allows proper description of many-body dynamic correlations, shows that the ideal transition at $T_{\mathrm{MCT}}$ can be delayed by retaining higher order density correlations in the MCT equations 14.

The importance of high order static correlations, hidden in the amorphous structure of the liquid, is particularly emphasized by frustration-based approaches to the glass transition [15-18. According to these theories, the phenomenology of glass formation arises from the competition between the growth of slow, correlated domains, characterized by some preferred local order, and frustration, which prevents these domains from percolating through the liquid. Despite some disagreement on the interpretation of the role of frustration [19, 20], these models indicate medium range order and structural correlations beyond the pair level as key features for understanding the dynamic behavior of glass-forming systems.

Computer simulations of several model glassy systems 21 27 and experiments on dense colloidal suspensions [28, 29] provide evidence for the existence of domains formed by preferred local structures and for their influence on the dynamics. Similar observations, albeit without explicit reference to the dynamics, emerge from recent $a b$ initio simulations and experiments on metallic glasses [30, 31. Furthermore, high order static correlations, named "point-to-set" correlations [32, have recently been revealed by simulations under amorphous boundary conditions and have been found to grow by decreasing temperature in a model supercooled liquid [33]. 


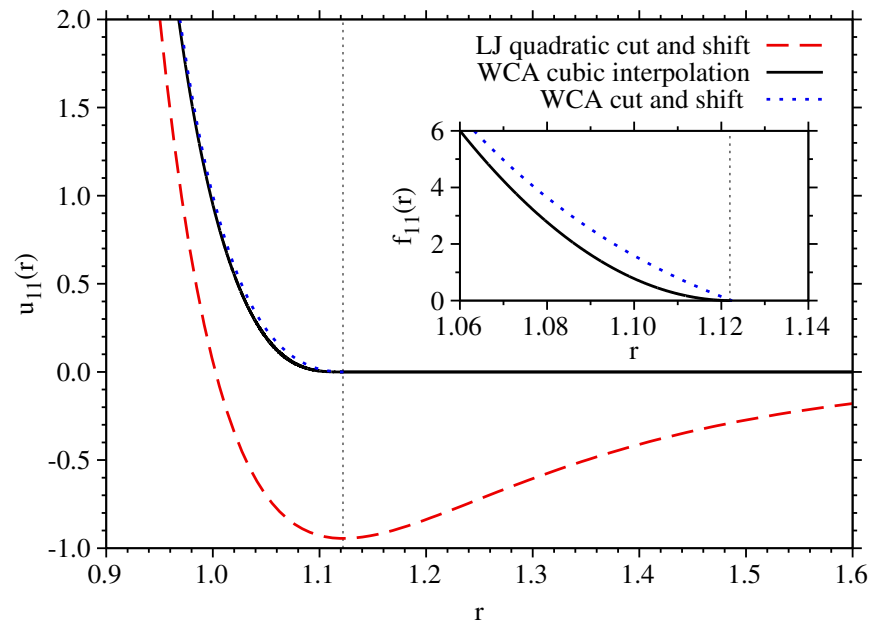

FIG. 1. Main panel: pair potentials between particles of species 1 used in this study: LJ (dashed line), WCAS (solid line), and WCA (dotted line). Inset: force between particles of species 1 for WCAS (solid line) and WCA (dotted line) potentials. In both panels, the dotted vertical line marks the distance $r=2^{1 / 6}$ corresponding to the minimum of the LJ potential.

In spite of these advances, there is still no general consensus on the connection between the structure and dynamics in supercooled liquids. In particular, dynamic facilitation models 34 provide an alternative and physically appealing description of the glassy dynamics in terms of purely kinetic constraints.

A clear-cut procedure to test the influence of manybody static correlations on the dynamics of glass-forming liquids emerges from recent work of Berthier and Tarjus [8, 35. These authors compared the pair structure and dynamics of two model glassy systems: the KobAndersen (KA) binary Lennard-Jones (LJ) mixture [1] and its Weeks-Chandler-Andersen (WCA) variant 36], in which the attractive part of the pair potential is truncated. Berthier and Tarjus found that, at fixed temperature and for sufficiently large density, the pair structure of the two models is almost identical, while the structural relaxation times can differ by orders of magnitude. Thus, direct comparison of LJ and WCA models offers an ideal benchmark to test the existence and the influence of static correlations beyond the pair level. Building on prior knowledge on the preferred local order of LJ mixtures [26], we will consider here structural indicators of increasing complexity - ranging from pair correlations, through angular distribution functions, to Voronoi tessellation - for selected LJ and WCA liquids and perform a crucial numerical experiment on the influence of structure on the dynamics of the models.

\section{METHODS}

We will consider two well-known models of glassforming liquids: the Kob-Andersen binary mixture [11] and the Wahnström binary mixture (WAHN) [37]. In the original models [11, 37, named herein KA-LJ and WAHN-LJ, particles interact through the LJ potential

$$
u_{\alpha \beta}(r)=4 \epsilon_{\alpha \beta}\left[\left(\frac{\sigma_{\alpha \beta}}{r}\right)^{12}-\left(\frac{\sigma_{\alpha \beta}}{r}\right)^{6}\right]
$$

where $\alpha, \beta=1,2$ are species indices. The values of the parameters $\sigma_{\alpha \beta}$ and $\epsilon_{\alpha \beta}$ are $\sigma_{12}=0.8 \sigma_{11}, \sigma_{22}=0.88 \sigma_{11}$, $\epsilon_{12}=1.5 \epsilon_{11}$, and $\epsilon_{22}=0.5 \epsilon_{11}$ for the KA mixture, and $\sigma_{12}=0.916 \sigma_{11}, \sigma_{22}=0.833 \sigma_{11}$, and $\epsilon_{22}=\epsilon_{12}=\epsilon_{11}$ for the WAHN mixture. The chemical compositions and mass rations are $x_{1}=1-x_{2}=0.8, m_{1} / m_{2}=1$ (for the KA mixture) and $x_{1}=x_{2}=0.5, m_{1} / m_{2}=2$ (for the WAHN mixture). The potentials are cut and shifted by a quadratic term 38 at $2.5 \sigma_{\alpha \beta}$ and $2.5 \sigma_{11}$ in $\mathrm{KA}$ and WAHN mixtures, respectively. In the following, we will use $\sigma_{11}, \epsilon_{11}$, and $\sqrt{m_{1} \sigma_{11}^{2} / \epsilon_{11}}$ as units of distance, energy, and time, respectively.

In addition, we study the corresponding WCA variants of the above mixtures. In the WCA models [36, 39], the interaction parameters $\sigma_{\alpha \beta}$ and $\epsilon_{\alpha \beta}$ and chemical compositions are unchanged but each of the pair potentials $u_{\alpha \beta}(r)$ is truncated and shifted so that the value at the minimum is zero 39. The WCA truncation of the attractive part of the potential is well-known from liquid state theories 39. We found, however, that this procedure leads to poor energy conservation during the long molecular dynamics simulations in the supercooled regime. To circumvent this problem, we employ here a smooth cut off scheme with cubic interpolation [40] to ensure continuity up to the second derivative of the potentials at the minimum $r_{c}=2^{1 / 6} \sigma_{\alpha \beta}$ of the LJ potential. Explicitly, the WCA smoothed potentials (WCAS) read

$$
u_{\alpha \beta}^{s}(r)= \begin{cases}u_{\alpha \beta}(r)+A_{\alpha \beta} & r<a_{\alpha \beta} \\ B_{\alpha \beta}\left(r_{c}-r\right)^{3} & a_{\alpha \beta}<r<r_{c} \\ 0 & r>r_{c}\end{cases}
$$

where $A_{\alpha \beta}$ and $B_{\alpha \beta}$ are determined to ensure continuity at $r=a$ and $r=r_{c}$. The parameters $a_{\alpha \beta}$ are adjusted for each pair $\alpha$ - $\beta$ so that $r_{c}=2^{1 / 6} \sigma_{\alpha \beta}$, and read $a_{11}=1.0269, a_{12}=0.8215, a_{22}=0.9038$ for the KA-WCAS mixture, and $a_{11}=1.0269, a_{12}=0.9473$, $a_{22}=0.8555$ for the WAHN-WCAS mixture. A comparison between LJ, WCA, and WCAS potentials for 1-1 pairs is shown in Fig. 1. In contrast to the WCA potential, the derivative of the force of the WCAS potential is continuous at $r_{c}$. In the inset, we highlight the difference between the WCA and the WCAS potentials in the $r \sim r_{c}$ region. As it will be clear in the following, this modification introduces some small differences in the thermodynamic and dynamic properties, but does not qualitatively alter the comparison with LJ models. In the following, we will mostly concentrate on the WCAS models and report 


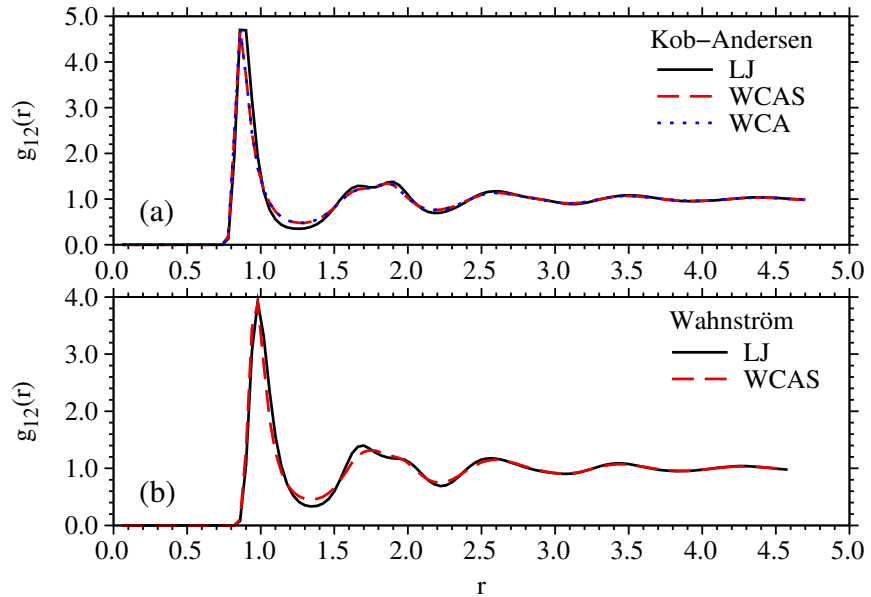

FIG. 2. Radial distribution functions $g_{12}(r)$ for (a) the KobAndersen mixture and (b) the Wahnström mixture. The state points considered are (a) $\rho=1.2, T=0.5$ and (b) $\rho=1.297$, $T=0.6$. In both panels, solid, dashed, and dotted lines indicate results for the LJ, WCAS, and WCA models, respectively. Error bars are smaller than the widths of the lines.

selected results for the original WCA models. All studied systems are composed of 1000 particles in a cubic box with periodic boundary conditions. Molecular dynamics simulations are performed in the NVT ensemble using the Nosé-Poincaré thermostat 41 with a mass parameter $Q=5.0$. The number density of the KA mixtures is $\rho=1.2$, while that of Wahnström mixtures is $\rho=1.297$. For the LJ and WCAS models, static and dynamic properties are averaged over up to six independent thermal histories. We find that the KA-WCAS mixture crystallizes more easily than the other systems [42]. A similar tendency to crystallize has been reported in Ref. 43 . for the KA-WCA model. Only the non-crystallizing samples are retained to perform the averages.

\section{RESULTS}

\section{A. Two-body and three-body static correlations}

To start the discussion, we analyze the pair structure of the present models. Figure 2 displays the radial distribution function $g_{12}(r)$ between unlike species in the KA mixtures (top panel) and WAHN mixtures (bottom panel). For each type of mixture, the results obtained are shown at a common temperature, representative of the slow dynamics regime of the LJ models. The pair structure appears essentially unaffected by the truncation of attractions, thus confirming the observations of recent simulation works 8, 44. This result holds for both WCA and WCAS models. Only a close inspection of the figures reveals that the first minima of $g_{12}(r)$ are slightly deeper in the LJ models, suggesting that the latter systems are effectively more supercooled. A similar effect is visible in

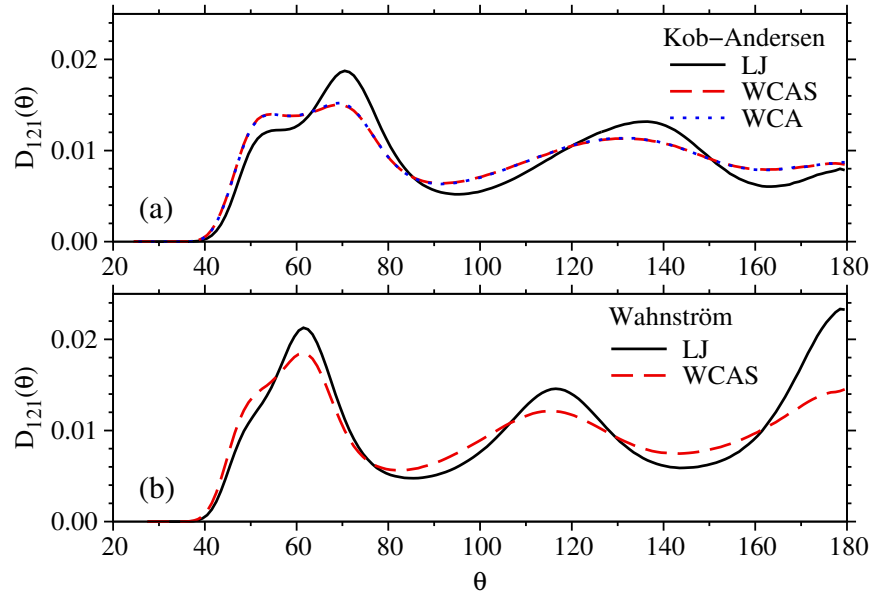

FIG. 3. Angular distribution functions $D_{121}(r)$ for (a) Kob-Andersen mixtures and (b) Wahnström mixtures. State points and lines are the same as in Fig. 2 Error bars are smaller than the widths of the lines.

the radial distribution functions reported by Pedersen et al. 44 for KA-LJ and KA-WCA mixtures.

At the temperatures considered in Fig. 2, the structural relaxation times of the LJ and WCA models differ by almost two orders of magnitude, as is evident from Ref. 8 and Figs. 4 and 5 (discussed in further detail below). Given the small differences observed in the pair structure, it is natural to ask whether this large variation in the dynamic properties is due to static correlations of higher order. In a first attempt to go beyond pair correlations, we calculate the bond-angle distribution functions $D_{\alpha \beta \gamma}(\theta)$ between triplets of neighboring particles of particles of species $\alpha, \beta$, and $\gamma$, where $\beta$ is the species of the central particle. Figure 3 shows the angular distribution functions $D_{121}(\theta)$ for the same state points considered in Fig. 2. Angular correlations reveal more clearly the structural differences between the LJ and WCA models. In KA mixtures, the sharp peaks in $D_{121}(\theta)$ around $\sim 70^{\circ}$ and the broad peak in the range $120^{\circ}-140^{\circ}$ reflect local arrangements corresponding to distorted twisted bi-capped prisms of large particles (species 1) centered around small particles (species 2) [26]. A comparison of the LJ and WCA data sets thus reveals that the KA-LJ mixture has a more pronounced local ordering than the KA-WCA mixture at the selected thermodynamic state. As in the case of $g_{12}(r)$, the difference between the WCA and WCAS models is negligible for this state point. A similar effect is visible for the WAHN mixture: the peaks in $D_{121}(\theta)$, located around $63^{\circ}, 116^{\circ}$, and $180^{\circ}$, are signatures of local icosahedral ordering, which appears more pronounced in the original LJ model than in the WCAS variant. Similar conclusions can be drawn from an analysis of the other angular distribution functions (not shown here) and are corroborated by an inspection of data at even lower temperature. We conclude that the structure of LJ and WCA systems differ more evidently at the level of three-body static correlations and that the increase 

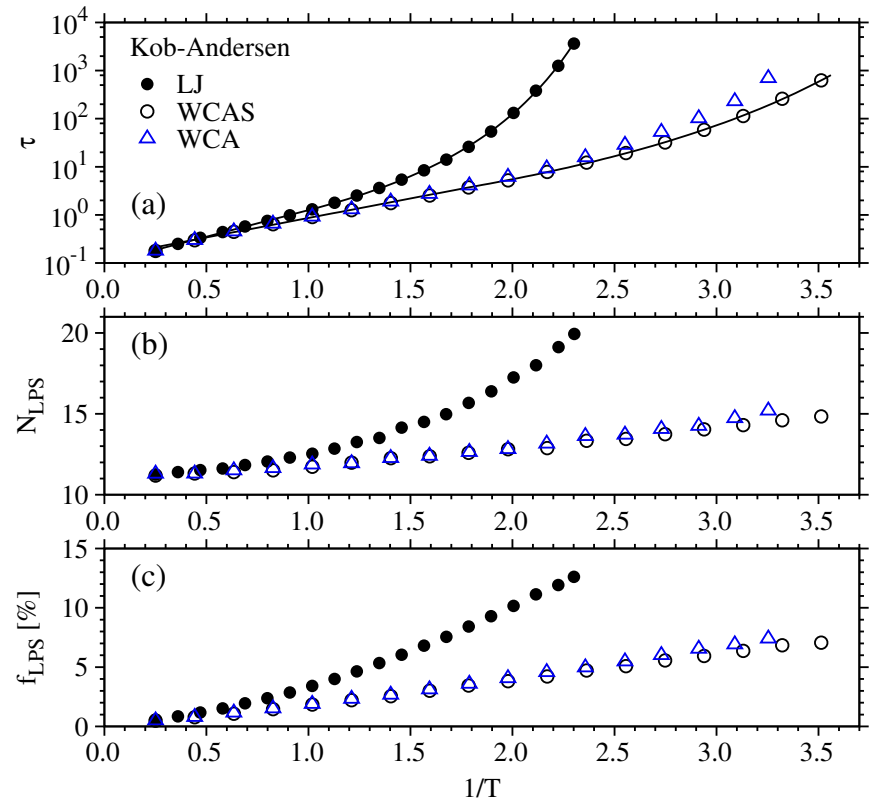

FIG. 4. (a) Structural relaxation times $\tau$ as a function of $1 / T$ for Kob-Andersen mixtures: LJ (filled circles), WCAS (open circles), and WCA (open triangles) models. The wave-vector considered for the calculation of $\tau$ is $k=7.0$. Fits to the modified VFT equation (Eq. 3) are shown as solid lines. (b) Average number $N_{\text {LPS }}$ of particles in LPS domains formed by $(0,2,8)$-polyhedra. Symbols have the same meaning as in (a). (c) Average fraction of particles of species 2 at the center of $(0,2,8)$ polyhedra as a function of $1 / T$. Symbols have the same meaning as in (a).

of local ordering upon switching on attractions correlate qualitatively with the increase of relaxation times.

\section{B. Locally preferred structures}

To render the connection between the local structure and dynamics explicit, we analyze the statistics of Voronoi polyhedra as a function of temperature. The Voronoi tessellation implicitly entails more complex static correlations (although, of course, it cannot be expressed as a multi-particle correlation function) and reveals the details of the particles' arrangements within the first coordination shell. Inspection of the spatial persistence of a given local structure provides information on extended structural correlations, i.e., medium range order. The protocol adopted here is the same as that used in a prior investigation of the local structure of binary LJ mixtures at constant pressure 26. We monitor the temperature dependence of the fraction of Voronoi polyhedra with a given signature $\left(n_{3}, n_{4}, \ldots\right)$, where $n_{i}$ is the number of faces of the polyhedron with a given number $i$ of vertices. We identify the locally preferred structure (LPS) of the liquid as the geometrical arrangement corresponding to the most frequent Voronoi polyhedron around particles of species 2 observed in the samples at
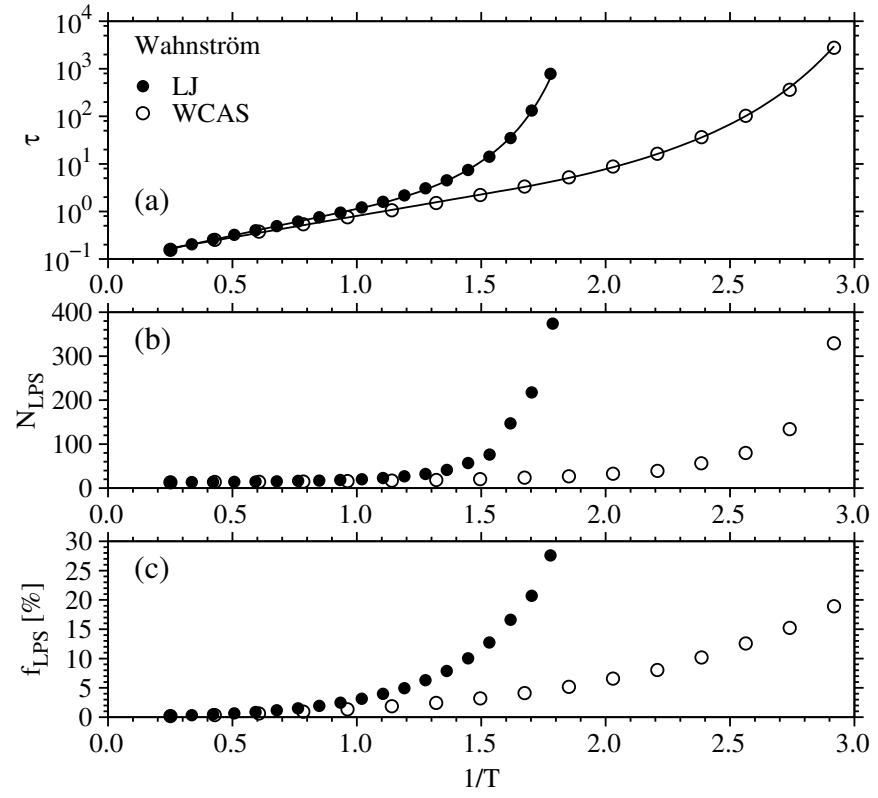

FIG. 5. Same as Fig. 4 but for Wahnström mixtures. For these systems, the LPS corresponds to $(0,0,12)$ polyhedra.

low temperature. This choice is based on the observation that in binary LJ mixtures it is easier to characterize local order around small particles [26, 45]. This is also consistent with a previous study of the KA-LJ mixture, which focused on the coordination polyhedra of large particles around the small ones [46]. The most frequent signatures of Voronoi polyhedra around particles of species 2 and 1 are reported in Table I and II] respectively. We find that the typical Voronoi polyhedra observed around particles of species 1 [such as $(0,2,8,4)$ or $(0,1,10,4)$ polyhedra] do not display evident symmetries and lack a clear structural identification. Furthermore, the corresponding percentages do not increase as sharply upon decreasing temperature as those calculated for Voronoi polyhedra around particles of species 2 . In the following, we will therefore base our analysis on the local structures observed around this latter type of particles. Understanding the nature of local order around large particles remains an open issue, which may need more refined methods to detect short and medium range order.

Our results confirm the observations of Ref. [26] at constant pressure: in the low temperature regime, $(0,2,8)$ and $(0,0,12)$ polyhedra around particles of species 2 constitute the dominant signatures in KA-LJ and WAHN-LJ mixtures, respectively. Thus, we identify the LPS of KALJ and WAHN-LJ as twisted bi-capped square prisms and icosahedra, respectively [26]. The identification is consistent with previous investigations on KA-LJ clusters [7] and with a recent simulation study on the bulk WAHN-LJ mixture 48. By applying the same procedure to the WCA and WCAS models, we find that the locally preferred structures remain the same as in the original LJ models. We observe, however, a systematic reduction 
TABLE I. Most frequent signatures of Voronoi polyhedra around particles of species 2 from instantaneous configurations and local minima of potential energy surface. Percentages are calculated with respect to the number of particles of species 2. The low temperature data set $\left(T=T_{l}\right)$ corresponds to the lowest available temperatures: $T=0.435(\mathrm{KA}-\mathrm{LJ}), T=0.285$ (KA-WCAS), $T=0.560$ (WAHN-LJ), and $T=0.343$ (WAHN-WCAS). The high temperature data set $\left(T \approx T^{*}\right)$ corresponds to temperatures close to the crossover temperature $T^{*}: T=0.983$ (KA-LJ), $T=0.627$ (KA-WCAS), $T=1.072(\mathrm{WAHN}-\mathrm{LJ})$, and $T=0.598$ (WAHN-WCAS).

\begin{tabular}{|c|c|c|c|c|c|c|c|c|}
\hline & \multicolumn{4}{|c|}{ Instantaneous configurations } & \multicolumn{4}{|c|}{ Local minima } \\
\hline & \multicolumn{2}{|c|}{$T=T_{l}$} & \multicolumn{2}{|c|}{$T \approx T^{*}$} & \multicolumn{2}{|c|}{$T=T_{l}$} & \multicolumn{2}{|c|}{$T \approx T^{*}$} \\
\hline & $\%$ & Signature & $\%$ & Signature & $\%$ & Signature & $\%$ & Signature \\
\hline \multirow[t]{4}{*}{ KA-LJ } & 12.6 & $(0,2,8)$ & 4.0 & $(0,2,8,1)$ & 19.9 & $(0,2,8)$ & 12.4 & $(0,2,8)$ \\
\hline & 8.3 & $(1,2,5,3)$ & 3.9 & $(1,2,5,3)$ & 7.2 & $(1,2,5,3)$ & 5.9 & $(1,2,5,3)$ \\
\hline & 5.6 & $(1,2,5,2)$ & 3.4 & $(0,2,8)$ & 6.8 & $(1,2,5,2)$ & 5.8 & $(1,2,5,2)$ \\
\hline & 5.1 & $(0,3,6)$ & 3.2 & $(0,4,4,3)$ & 6.7 & $(0,3,6)$ & 5.3 & $(0,3,6,1)$ \\
\hline \multirow[t]{4}{*}{ KA-WCAS } & 7.1 & $(0,2,8)$ & 3.9 & $(0,2,8,1)$ & 8.4 & $(0,2,8)$ & 5.2 & $(0,2,8)$ \\
\hline & 5.7 & $(1,2,5,3)$ & 3.5 & $(1,2,5,3)$ & 5.4 & $(1,2,5,3)$ & 4.1 & $(1,2,5,3)$ \\
\hline & 4.7 & $(0,2,8,1)$ & 3.4 & $(0,3,6,3)$ & 4.5 & $(1,2,5,2)$ & 4.0 & $(0,4,4,3)$ \\
\hline & 3.7 & $(0,4,4,3)$ & 3.1 & $(0,4,4,3)$ & 4.3 & $(0,2,8,1)$ & 3.9 & $(1,2,5,2)$ \\
\hline \multirow[t]{4}{*}{ WAHN-LJ } & 27.4 & $(0,0,12)$ & 7.0 & $(0,3,6,4)$ & 32.7 & $(0,0,12)$ & 10.9 & $(0,0,12)$ \\
\hline & 9.0 & $(0,2,8,2)$ & 5.2 & $(0,2,8,2)$ & 10.0 & $(0,2,8,2)$ & 10.6 & $(0,2,8,2)$ \\
\hline & 7.7 & $(0,1,10,2)$ & 3.5 & $(0,1,10,2)$ & 8.3 & $(0,1,10,2)$ & 9.7 & $(0,3,6,4)$ \\
\hline & 6.0 & $(0,3,6,4)$ & 3.0 & $(0,3,6,3)$ & 6.5 & $(0,3,6,4)$ & 7.3 & $(0,1,10,2)$ \\
\hline \multirow[t]{4}{*}{ WAHN-WCAS } & 18.9 & $(0,0,12)$ & 7.7 & $(0,3,6,4)$ & 20.0 & $(0,0,12)$ & 8.5 & $(0,3,6,4)$ \\
\hline & 9.1 & $(0,2,8,2)$ & 6.4 & $(0,2,8,2)$ & 9.8 & $(0,2,8,2)$ & 7.9 & $(0,2,8,2)$ \\
\hline & 7.2 & $(0,3,6,4)$ & 4.3 & $(0,1,10,2)$ & 7.4 & $(0,3,6,4)$ & 5.8 & $(0,0,12)$ \\
\hline & 6.9 & $(0,1,10,2)$ & 4.1 & $(0,0,12)$ & 6.6 & $(0,1,10,2)$ & 4.7 & $(0,1,10,2)$ \\
\hline
\end{tabular}

TABLE II. Same as Table I but for Voronoi polyhedra around particles of species 1. Percentages are calculated with respect to the number of particles of species 1 .

\begin{tabular}{|c|c|c|c|c|c|c|c|c|}
\hline & \multicolumn{4}{|c|}{ Instantaneous configurations } & \multicolumn{4}{|c|}{ Local minima } \\
\hline & \multicolumn{2}{|c|}{$T=T_{l}$} & \multicolumn{2}{|c|}{$T \approx T^{*}$} & \multicolumn{2}{|c|}{$T=T_{l}$} & \multicolumn{2}{|c|}{$T \approx T^{*}$} \\
\hline & $\%$ & Signature & $\%$ & Signature & $\%$ & Signature & $\%$ & Signature \\
\hline \multirow[t]{4}{*}{ KA-LJ } & 7.8 & $(0,2,8,4)$ & 4.7 & $(0,2,8,4)$ & 8.7 & $(0,2,8,4)$ & 7.2 & $(0,2,8,4)$ \\
\hline & 5.7 & $(0,2,8,5)$ & 3.2 & $(0,3,6,5)$ & 6.6 & $(0,2,8,5)$ & 5.1 & $(0,2,8,5)$ \\
\hline & 5.0 & $(0,3,6,6)$ & 3.0 & $(0,3,6,4)$ & 5.3 & $(0,3,6,6)$ & 4.1 & $(0,3,6,6)$ \\
\hline & 4.4 & $(0,3,6,5)$ & 2.9 & $(0,2,8,5)$ & 4.8 & $(0,1,10,4)$ & 4.1 & $(0,1,10,2)$ \\
\hline \multirow[t]{4}{*}{ KA-WCAS } & 7.4 & $(0,2,8,4)$ & 5.2 & $(0,2,8,4)$ & 7.5 & $(0,2,8,4)$ & 6.1 & $(0,2,8,4)$ \\
\hline & 5.0 & $(0,2,8,5)$ & 3.5 & $(0,3,6,5)$ & 5.1 & $(0,2,8,5)$ & 3.9 & $(0,2,8,5)$ \\
\hline & 4.7 & $(0,3,6,6)$ & 3.2 & $(0,3,6,4)$ & 4.9 & $(0,3,6,6)$ & 3.8 & $(0,3,6,5)$ \\
\hline & 4.5 & $(0,3,6,5)$ & 3.2 & $(0,3,6,6)$ & 4.4 & $(0,3,6,5)$ & 3.8 & $(0,3,6,6)$ \\
\hline \multirow[t]{4}{*}{ WAHN-LJ } & 8.0 & $(0,1,10,4)$ & 4.7 & $(0,2,8,4)$ & 9.5 & $(0,1,10,4)$ & 7.4 & $(0,2,8,5)$ \\
\hline & 7.1 & $(0,2,8,5)$ & 3.7 & $(0,2,8,5)$ & 8.5 & $(0,2,8,5)$ & 6.8 & $(0,2,8,4)$ \\
\hline & 6.3 & $(0,2,8,4)$ & 3.2 & $(0,3,6,6)$ & 6.7 & $(0,2,8,4)$ & 5.4 & $(0,1,10,4)$ \\
\hline & 4.7 & $(0,1,10,3)$ & 3.0 & $(0,3,6,5)$ & 5.2 & $(0,1,10,3)$ & 4.6 & $(0,3,6,6)$ \\
\hline \multirow[t]{4}{*}{ WAHN-WCAS } & 7.6 & $(0,2,8,5)$ & 5.8 & $(0,2,8,4)$ & 8.0 & $(0,2,8,5)$ & 6.3 & $(0,2,8,4)$ \\
\hline & 6.9 & $(0,2,8,4)$ & 4.8 & $(0,2,8,5)$ & 6.9 & $(0,2,8,4)$ & 5.8 & $(0,2,8,5)$ \\
\hline & 6.8 & $(0,1,10,4)$ & 3.8 & $(0,3,6,6)$ & 6.8 & $(0,1,10,4)$ & 4.4 & $(0,3,6,6)$ \\
\hline & 4.6 & $(0,1,10,3)$ & 3.4 & $(0,3,6,5)$ & 4.5 & $(0,1,10,3)$ & 3.6 & $(0,1,10,4)$ \\
\hline
\end{tabular}


of the fraction of LPS upon truncating the attractions. This effect will be discussed in further detail below.

As a general rule, the fraction $f_{\text {LPS }}$ of particles of species 2 at the center of a LPS increases with decreasing temperature [26]. The growth of $f_{\text {LPS }}$ reflects the formation of slow, long-lived clusters of neighboring LPS [26]. A calculation of the self intermediate scattering functions filtered according to the pertinent Voronoi polyhedra, shows that the typical relaxation times of particles at the center of LPSs are up to 10 times larger than those outside LPSs 26]. In the following, we will refer to these clusters as "LPS domains", which are defined as groups of particles sitting either at the center or on the vertices of face-sharing polyhedra with the signature of the LPS. The average number of particles forming an LPS domain will be denoted by $N_{\text {LPS }}$, which is a measure of the spatial extension over which the liquid adopts the same preferred local structure 49. In Figs. 4(b) and 5(b) we show $N_{\text {LPS }}$ as a function of $1 / T$ for the KA and WAHN models, respectively. To facilitate a comparison with previous work [26], we include the temperature dependence of $f_{\text {LPS }}$ in Figs. 4(c) and 5(c). Note that, while $f_{\text {LPS }}$ is evaluated with respect to particles of species 2 , both species of particles contribute to the size $N_{\text {LPS }}$ of LPS domains. Both $N_{\text {LPS }}$ and $f_{\text {LPS }}$ increase in a similar fashion as $T$ decreases, although with slightly different functional forms. The growth of LPS domains is particularly dramatic in WAHN mixtures, which develop a strong icosahedral order upon supercooling. By contrast, the size of the domains formed by prismatic structures in $\mathrm{KA}$ models is relatively small (20-30 particles). Nonetheless, the structural evolution in all the systems studied follow qualitatively similar patterns.

\section{Connection between structure and dynamics}

To illustrate the connection to the dynamics of the models, we now study the temperature dependence of the structural relaxation times $\tau$. The latter are defined by the condition $F_{s}(k=7, \tau)=1 / e$, where $F_{s}(k, t)$ is the self intermediate scattering function averaged over all particles. The relaxation times have been fitted by the following modified Vogel-Fulcher-Tammann (VFT) equation 26 ]

$$
\tau(T)= \begin{cases}\tau_{\infty} \exp \left[E_{\infty} / T\right] & T>T^{*} \\ \tau_{\infty}^{\prime} \exp \left[\frac{1}{K\left(T / T_{0}-1\right)}\right] & T<T^{*}\end{cases}
$$

where

$$
\tau_{\infty}^{\prime}=\tau_{\infty} \exp \left[E_{\infty} / T^{*}-\frac{1}{K\left(T^{*} / T_{0}-1\right)}\right]
$$

Equation (3) ensures a smooth crossover around $T^{*}$ between the Arrhenius law at high $T$ and the VFT equation at low $T$, accounting for the super-Arrhenius dependence of the relaxation times. Figures 4 (a) and 5 (a) display $\tau$ as a function of $1 / T$ for the KA and WAHN mixtures, respectively, together with the corresponding fits to Eq. (3). Figure 4(a) also includes results for the KA-WCA mixture obtained using the original cut and shift at the minimum of the potentials, as in previous works [8, 35, 36]. The latter data set is in good agreement with the results obtained in Refs. 8, 35 for a similar wave-vector $(k=7.21)$. At sufficiently low temperature, however, non-negligible deviations appear with respect to the KAWCAS mixture. This discrepancy may be attributed to the modification induced by the smooth cut off employed in this work. The comparison between the LJ and WCAS models, however, remains qualitatively unaffected and confirms the general conclusions of Ref. [8, 35].

A comparison of the LPS analysis and relaxation times data reveals a striking relationship between structure and dynamics. The increase of $\tau$ below the crossover temperature $T^{*}$ correlates to the increase in size of the LPS domains. This connection is particularly evident for the two WAHN mixtures, in which the increase of icosahedral order upon decreasing $T$ is very sharp. Our results reveal that the large difference in the dynamic behavior between the LJ and WCA models reflects different stages of the evolution of the local structure on the way to glass formation - an effect that is barely visible at the level of pair correlations. It should also be noted that the size of the LPS domains at low temperature is slightly larger in the WCA than in the WCAS models, which is consistent with the discrepancy in relaxation times observed above.

A remark on the nature of local order in these models is in order. It has been shown recently that the WAHN-LJ mixture can phase-separate and then partially crystallize in a complex crystal structure that accommodates distorted icosahedral geometries 48. The LPS observed in the liquid is thus analogous to the typical local structure of the underlying crystal, which is at odds with the paradigm of the frustration-limited domains theory [16]. The situation is less clear in the case of the KA-LJ mixture, for which an unambiguous identification of the crystalline phase is lacking. Previous studies [50, 51, have shown that, for chemical compositions close to the one of the original model, stable crystals either have $\mathrm{CsCl}$ symmetry or are composed of a mixture of fcc and hcp structures of large particles. Interestingly, we found that the KA-WCAS model can crystallize into a fcc lattice of large particles. In this model crystallization is associated with a sudden drop in the fraction of $(0,2,8)$ polyhedra and a rapid increase of $(0,4,4,6)$ polyhedra centered around particles of species 1 . This reveals a potential mismatch between the LPS of the liquid and the typical local structure of the crystal. The question of whether the locally preferred structure should coincide with the structure of the underlying crystal [19, 20] certainly deserves further investigations. 


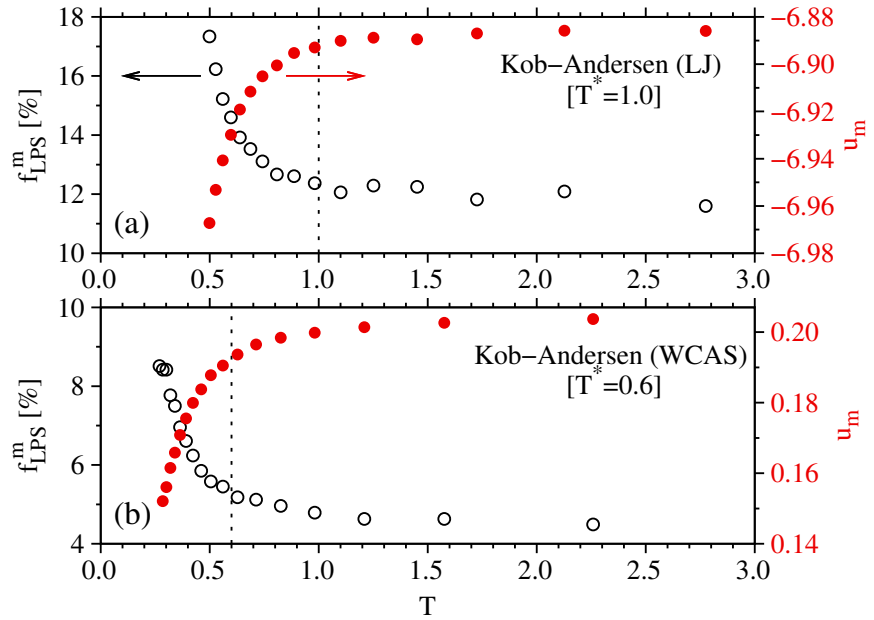

FIG. 6. Average fraction $f_{\mathrm{LPS}}^{m}$ of LPS in local minima (open circles, left axis) and average potential energy $u_{m}$ of local minima (filled circles, right axis) as a function of $T$ for KobAndersen mixtures: (a) LJ model and (b) WCAS model. The dynamic crossover temperatures $T^{*}$ obtained from fits to Eq. (3) are indicated as vertical dotted lines.

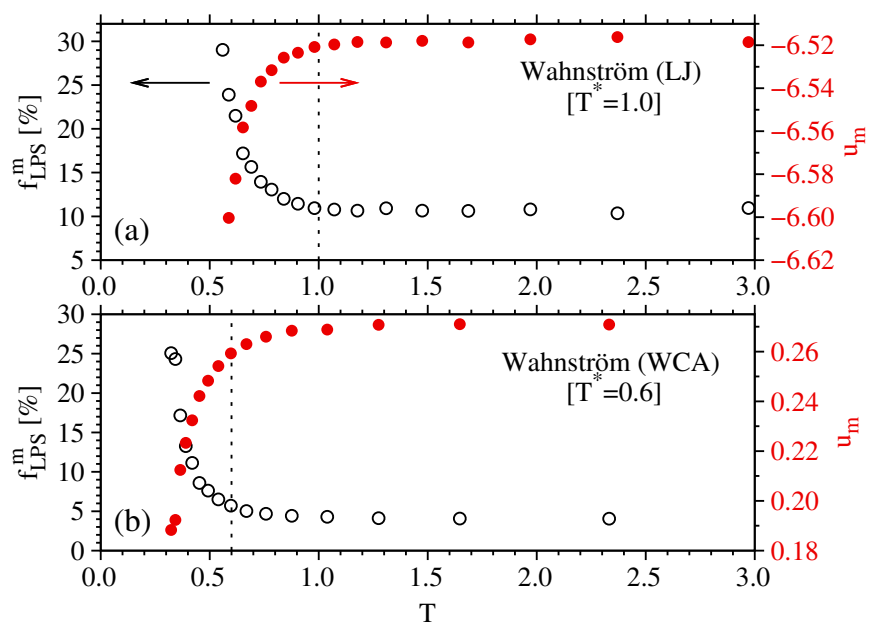

FIG. 7. Same as Fig. 6 but for Wahnström mixtures.

\section{Connections between structure, potential energy landscape and fragility}

The connection between the growth of LPS domains and slow dynamics is further corroborated by the analysis of the potential energy landscape (PEL). It is wellknown that the appearance of super-Arrhenius behavior and non-exponential relaxation around the so-called onset temperature $T_{O}$ coincides with a sharp change in the properties of the local minima of the PEL explored by system [52. In fact, the average energy $u_{m}(T)$ of local minima remains almost constant at high $T$ and starts decreasing rapidly below $T_{O}$. Figures 6 and 7 display $u_{m}$ and the fraction $f_{\mathrm{LPS}}^{m}$ of particles of species 2 at the center of the LPSs evaluated for local minima of the PEL as a function of temperature for the KA and WAHN mixtures, respectively. We note that the values of $T^{*}$ obtained from Eq. 3 are only slightly larger than the onset temperatures estimated from the appearance of two-step, non-exponential relaxation in the dynamic correlation functions, and are consistent, at least for KA mixtures, with the values of $T_{O}$ reported in Ref. [53.

Strikingly, the onset of slow dynamics, indicated by the drop in $u_{m}$, always correlates to a sharp increase of $f_{\mathrm{LPS}}^{m}$. We remark that the percentages of other, less frequent signatures of Voronoi polyhedra do not increase as sharply across $T^{*}$, although some of them do display some change upon decreasing $T$ (see Table I). We thus attribute the onset of slow dynamics in the models studied herein to the growth of structural correlations. We also note that landscape approaches based on high-order stationary points of the potential energy surface [54 may provide a complementary view on our results. We found in fact that particles at the center of LPSs participate less to unstable modes of saddle points [55 57. Establishing a clear relationship between the growth of LPSs and the disappearance of unstable directions in the landscape is an interesting open issue that is left for future investigation.

The present findings ostensibly indicate that the drop in $u_{m}$ is connected to the formation of energetically favored structures. This is consistent with the observation that the potential energy associated with the LPS is typically lower than that of other structures [55]. In general, however, specific local structures may be favored also for non-energetic reasons, such as more efficient packing or non-trivial entropic effects (e.g., favorable arrangements of interlocking LPS). An interesting example of this competition is provided by a model of a NiY alloy based on LJ interactions [45, for which the LPS - a capped trigonal prismatic structure - corresponds to a Voronoi polyhedron having the smallest volume but not the lowest potential energy 55. Successful attempts to account for this complex interplay at the mean-field level in the one-component LJ liquid [58] and in a soft-sphere mixture [59, 60] must also be acknowledged.

To set forth the present results in a more compact fashion, we now plot $\tau$ as a function of $N_{\text {LPS }}$ (see Fig. 8), thereby making the temperature dependence implicit. This representation of the data allows us to illustrate more clearly several system-specific aspects of the relationship between the structure and dynamics. As expected, we find that the increase of $\tau$ correlates to that of $N_{\text {LPS }}$. However, the spatial extension of LPS domains at fixed relaxation times increases systematically with the fragility $K$ of the model, which is estimated from fitting the relaxation times to Eq. 3. In the case of the more fragile WAHN mixtures, the increase of relaxation times is evidently dictated by the growth of LPS domains. Over the same range of $\tau$, the less fragile KA mixtures show a weaker structural change, indicating that other effects, such as dynamic facilitation, may also be playing an important role. The overall trend of variation is consistent 


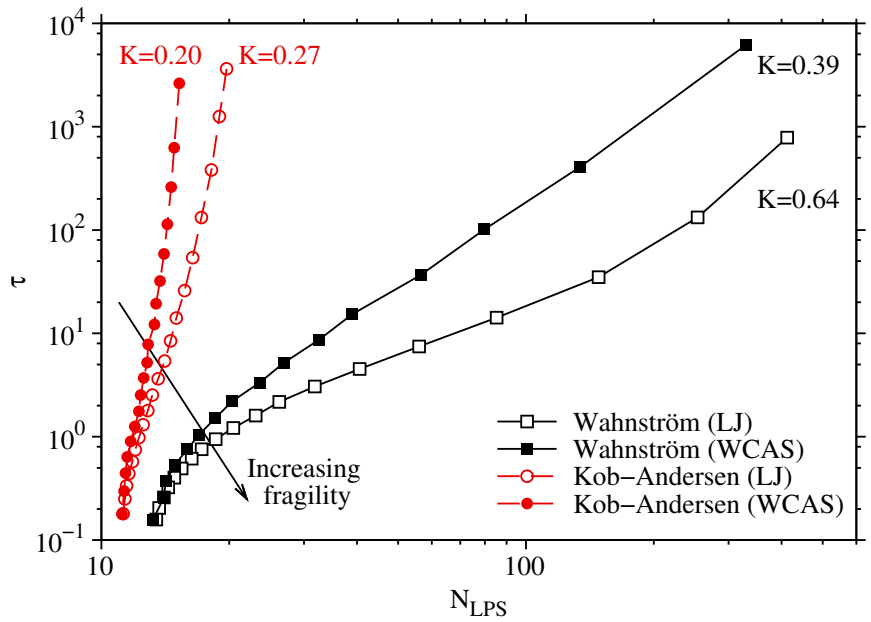

FIG. 8. Relaxation times $\tau$ as a function of the average number $N_{\text {LPS }}$ of particles in the LPS domains. The corresponding fragility indices $K$ of the models, obtained from fits to the modified VFT equation [Eq. [3] ], are also indicated.

with the correlation between the fragility and thermal rate of growth of LPSs put forth in Ref. [26] and suggests that the impact of static correlations on the dynamics should be more pronounced the more fragile the liquid. We also note that the inclusion of attractions tends to increase $N_{\text {LPS }}$ at fixed relaxation times. This stabilization effect is in qualitative agreement with recent observations on LJ and WCA fluids close to the triple point 61.

\section{CONCLUSIONS}

We have performed a crucial test on the dynamic role of static correlations in glass-forming liquids by comparing two well-studied LJ mixtures and their corresponding purely repulsive variants. Truncation of the attractive part of the potential considerably shifts the glass tran- sition to lower temperatures and reduces the fragility of the liquid, but does not alter the pair structure significantly 35. These phenomena have been explained by resorting to indicators revealing more complex structural correlations. Building on prior work [26], we have identified correlated domains formed by locally preferred structures. We have found that the growth, by decreasing the temperature, of LPS domains is tightly connected to the onset of the slow dynamics regime. Furthermore, an analysis of LPS domains has allowed us to clearly distinguish the different dynamic behaviors of the LJ and WCAS models in terms of their structure. In retrospect, these results suggest that even small differences that are discernible at the level of pair correlations, may reflect substantially different stages of the structural evolution of a supercooled liquid and be associated with very different dynamic regimes. A solution of the MCT equations for the dynamic correlation functions - using two-body static correlations as input - only partially accounts for the different dynamic behaviors of the LJ and WCA models [8, 62. Thus, proper inclusion of many-body static correlations in theories of the glass transition based on structural information seems crucial for a correct description of the dynamics in fragile glass-formers. This suggests revisiting and extending previous attempts [9] along these lines based on mode-coupling theory. Investigations of high order static correlations extracted from simulations under amorphous boundary conditions 33, using patch repetition 63] or order mining methods [64, as well as implementation of alternative methods for LPS determination [28, 65], may provide further clues to improve our theoretical understanding of the glass transition.

\section{ACKNOWLEDGMENTS}

Useful discussions with L. Berthier, W. Kob, and G. Pastore are acknowledged. D.C. acknowledge partial financial support by the Austrian Science Found (FWF) under Proj. No. P19890-N16.
[1] W. Götze, J. Phys.: Condens. Matter 11, A1 (1999).

[2] M. Nauroth and W. Kob, Phys. Rev. E 55, 657 (1997).

[3] S. P. Das, Reviews of Modern Physics 76, 785 (2004).

[4] Y. Brumer and D. R. Reichman, Phys. Rev. E 69, 041202 (2004).

[5] A. Andreanov, G. Biroli, and J.-P. Bouchaud, EPL 88, 16001 (2009).

[6] A. Ikeda and K. Miyazaki, Phys. Rev. Lett. 104, 255704 (2010).

[7] V. Lubchenko and P. G. Wolynes, Ann. Rev. Phys. Chem. 58, 235 (2007).

[8] L. Berthier and G. Tarjus, Phys. Rev. E 82, 031502 (2010).

[9] W. Kob, M. Nauroth, and F. Sciortino, J. Non-Cryst. Solids 307, 181 (2002).
[10] B. W. H. van Beest, G. J. Kramer, and R. A. van Santen, Phys. Rev. Lett. 64, 1955 (1990).

[11] W. Kob and H. C. Andersen, Phys. Rev. E 51, 4626 (1995).

[12] G. Szamel, Phys. Rev. Lett. 90, 228301 (Jun 2003).

[13] J. Wu and J. Cao, Phys. Rev. Lett. 95, 078301 (2005).

[14] P. Mayer, K. Miyazaki, and D. R. Reichman, Phys. Rev. Lett. 97, 095702 (2006).

[15] D. Kivelson, S. A. Kivelson, X. Zhao, Z. Nussinov, and G. Tarjus, Physica A. 219, 27 (1995).

[16] G. Tarjus, D. Kivelson, and P. Viot, J. Phys.: Condens. Matter 12, 6497 (2000).

[17] H. Tanaka, J. Non-Cryst. Solids 351, 3371 (2005).

[18] H. Tanaka, J. Non-Cryst. Solids 351, 3385 (2005).

[19] F. Sausset and G. Tarjus, Phys. Rev. Lett. 100, 099601 (2008). 
[20] T. Kawasaki, T. Araki, and H. Tanaka, Phys. Rev. Lett. 100, 099602 (2008).

[21] T. Tomida and T. Egami, Phys. Rev. B 52, 3290 (1995).

[22] M. Dzugutov, S. I. Simdyankin, and F. H. M. Zetterling, Phys. Rev. Lett. 89, 195701 (2002).

[23] T. S. Jain and J. de Pablo, J. Chem. Phys. 122, 174515 (2005).

[24] H. Shintani and H. Tanaka, Nature Physics 2, 200 (2006).

[25] I. Ladadwa and H. Teichler, Phys. Rev. E 73, 031501 (2006).

[26] D. Coslovich and G. Pastore, J. Chem. Phys. 127, 124504 (2007).

[27] F. Sausset and G. Tarjus, Phys. Rev. Lett. 104, 065701 (2010).

[28] C. P. Royall, S. R. Williams, T. Ohtsuka, and H. Tanaka, Nature Mater. 7, 556 (2008).

[29] H. Tanaka, T. Kawasaki, H. Shintani, and K. Watanabe, Nature Mater. 9, 324 (2010).

[30] T. Fujita, K. Konno, W. Zhang, V. Kumar, M. Matsuura, A. Inoue, T. Sakurai, and M. W. Chen, Phys. Rev. Lett. 103, 075502 (2009).

[31] A. Hirata, P. Guan, T. Fujita, Y. Hirotsu, A. Inoue, A. R. Yavari, T. Sakurai, and M. Chen, Nat Mater 10, 28 (2011).

[32] A. Montanari and G. Semerjian, J. Stat. Phys. 125, 23 (2006).

[33] G. Biroli, J.-P. Bouchaud, A. Cavagna, T. S. Grigera, and P. Verrocchio, Nat. Phys. 4, 771 (2008).

[34] J. P. Garrahan and D. Chandler, Proc. Natl. Acad. Sci. 100, 9710 (2003).

[35] L. Berthier and G. Tarjus, Phys. Rev. Lett. 103, 170601 (2009).

[36] D. Chandler, J. P. Garrahan, R. L. Jack, L. Maibaum, and A. C. Pan, Phys. Rev. E 74, 051501 (2006).

[37] G. Wahnström, Phys. Rev. A 44, 3752 (1991).

[38] S. D. Stoddard and J. Ford, Phys. Rev. A 8, 1504 (1973).

[39] J. D. Weeks, D. Chandler, and H. C. Andersen, J. Chem. Phys. 54, 5237 (1971).

[40] T. S. Grigera, A. Cavagna, I. Giardina, and G. Parisi, Phys. Rev. Lett. 88, 055502 (2002).

[41] S. Nosé, J. Phys. Soc. Jap. 70, 75 (2001).

[42] In this case, 4 samples out 6 crystallized around $T \sim 0.3$.

[43] S. Toxvaerd, U. R. Pedersen, T. B. Schroder, and J. C. Dyre, J. Chem. Phys. 130, 224501 (2009).
[44] U. R. Pedersen, T. B. Schrøder, and J. C. Dyre, Phys. Rev. Lett. 105, 157801 (2010).

[45] R. G. D. Valle, D. Gazzillo, R. Frattini, and G. Pastore, Phys. Rev. B 49, 12625 (1994).

[46] J. R. Fernández and P. Harrowell, J. Phys. Chem. B 108, 6850 (2004).

[47] J. P. K. Doye, A. A. Louis, I.-C. Lin, L. R. Allen, E. G. Noya, A. W. Wilber, H. C. Kok, and R. Lyus, Phys. Chem. Chem. Phys. 9, 2197 (2007).

[48] U. R. Pedersen, T. B. Schrøder, J. C. Dyre, and P. Harrowell, Phys. Rev. Lett. 104, 105701 (2010).

[49] Note that this correlation does not require neighboring LPS to be oriented in a similar fashion, provided a preferred direction of the LPS could be defined.

[50] J. R. Fernández and P. Harrowell, J. Phys. Chem. B 108, 6850 (2004)

[51] L.-C. Valdes, F. Affouard, M. Descamps, and J. Habasaki, J. Chem. Phys. 130, 154505 (2009).

[52] S. Sastry, P. G. Debenedetti, and F. H. Stillinger, Nature 393, 554 (1998).

[53] Y. S. Elmatad, D. Chandler, and J. P. Garrahan, The Journal of Physical Chemistry B 114, 17113 (2010).

[54] T. Grigera, A. Cavagna, I. Giardina, and G. Parisi, Phys. Rev. Lett. 88, 055502 (2002).

[55] D. Coslovich, Ph.D. thesis, Universitá degli Studi di Trieste (2008).

[56] D. Coslovich and G. Pastore, J. Chem. Phys. 127, 124505 (2007).

[57] D. Coslovich, unpublished.

[58] S. Mossa and G. Tarjus, J. Chem. Phys. 119, 8069 (2003).

[59] H. G. E. Hentschel, V. Ilyin, N. Makedonska, I. Procaccia, and N. Schupper, Phys. Rev. E 75, 050404(R) (2007).

[60] E. Lerner, I. Procaccia, and J. Zylberg, Phys. Rev. Lett. 102, 125701 (2009).

[61] J. Taffs, A. Malins, S. R. Williams, and C. P. Royall, J. Chem. Phys. 133, 244901 (2010).

[62] T. Voigtmann, Phys. Rev. Lett. 101, 095701 (2008).

[63] J. Kurchan and D. Levine, J. Phys. A: Math. Theor. 44, 035001 (2011)

[64] X. W. Fang, C. Z. Wang, Y. X. Yao, Z. J. Ding, and K. M. Ho, Phys. Rev. B 82, 184204 (2010).

[65] S. Mossa and G. Tarjus, J. Non-Cryst. Solids 352, 4847 (2006). 